\title{
Stem cell biology and regenerative medicine for neonatal lung diseases
}

\author{
Martin Kang ${ }^{1}$ and Bernard Thébaud ${ }^{2}$
}

Lung diseases remain one of the main causes of morbidity and mortality in neonates. Cell therapy and regenerative medicine have the potential to revolutionize the management of life-threatening and debilitating lung diseases that currently lack effective treatments. Over the past decade, the repair capabilities of stem/progenitor cells have been harnessed to prevent/rescue lung damage in experimental neonatal lung diseases. Mesenchymal stromal cells and amnion epithelial cells exert pleiotropic effects and represent ideal therapeutic cells for bronchopulmonary dysplasia, a multifactorial disease. Endothelial progenitor cells are optimally suited to promote lung vascular growth and attenuate pulmonary hypertension in infants with congenital diaphragmatic hernia or a vascular bronchopulmonary dysplasia phenotype. Induced pluripotent stem cells (iPSCs) are one of the most exciting breakthroughs of the past decade. Patientspecific iPSCs can be derived from somatic cells and differentiated into any cell type. IPSCS can be capitalized upon to develop personalized regenerative cell products for surfactant protein deficiencies - lethal lung disorders without treatment-that affect a single gene in a single cell type and thus lend themselves to phenotype-specific cell replacement. While the clinical translation has begun, more needs to be learned about the biology of these repair cells to make this translation successful.

\section{LIFE-THREATENING AND DEBILITATING LUNG DISEASES OF THE NEWBORN THAT LACK EFFECTIVE THERAPIES}

A dvances in perinatal care have markedly improved neonatal survival over the past 50 years (1). Because the lung is one of the last organs to mature, and because of the marked changes that occur in cardiorespiratory transition at birth, neonatal respiratory complications are still common. Regionalization, antenatal steroids and antibiotics, improved resuscitation strategies, exogenous surfactant, and new ventilation approaches have contributed to improved respiratory outcomes. However, a subset of life-threatening and debilitating lung diseases still lacks effective therapies.
Bronchopulmonary dysplasia (BPD), the chronic lung disease of prematurity, remains the main complication of extreme preterm birth and is associated with adverse neurodevelopmental outcome, retinopathy of prematurity, sepsis, and long-term respiratory complications into adulthood (2). BPD is a multifactorial disease impeding normal lung growth as early as in the late canalicular stage of development. The aberrant repair process leads to dysplastic lung growth with dysfunctional small conducting airways, large and simplified alveoli, fewer and/or maldistributed blood vessels, and altered extracellular matrix.

A subset of infants with BPD may display a more vascular phenotype (3), making them more prone to develop pulmonary hypertension $(\mathrm{PH})$, which doubles their risk of death (4). PH also complicates congenital diaphragmatic hernia $(\mathrm{CDH})$, a common congenital malformation $(1 / 2,000$ live births) with a high mortality rate $(\sim 50 \%)$. Although inhaled nitric oxide has simplified the management of neonatal $\mathrm{PH}, 50 \%$ fail to respond. Among survivors, there is an emerging pattern of late and chronic $\mathrm{PH}$ extending beyond the immediate neonatal period that significantly worsens the prognosis $(5,6)$. The reduction of the lung vascular bed may render any treatment strategy aiming at vasodilatation only futile.

Surfactant protein deficiencies cause severe respiratory distress at birth and are lethal or lead to an underestimated $10 \%$ of all childhood interstitial lung diseases (7). Lung transplantation is the only life-saving option, but is rarely available and carries high risks of mortality and morbidity.

Novel insights into stem cell biology over the past decade have unraveled the regenerative potential of stem cells and sparked the development of a panoply of disease-specific cell-based therapies that offer the promise of personalized regenerative cell products to substantially improve the outcome of these life-threatening and debilitating lung diseases.

\section{A VERY BRIEF HISTORY OF STEM CELLS}

"At the time, you don't think you are going to do something historic". Dr James Till, co-discoverer of stem cells

\footnotetext{
'Ottawa Hospital Research Institute, Sinclair Centre for Regenerative Medicine, Ottawa, Ontario, Canada; ${ }^{2}$ Division of Neonatology, Department of Pediatrics, Children's Hospital of Eastern Ontario, Ottawa, Ontario, Canada. Correspondence: Bernard Thébaud (bthebaud@toh.on.ca)

Received 7 June 2017; accepted 18 August 2017; advance online publication 18 October 2017. doi:10.1038/pr.2017.232
} 


\section{Review | Kang and Thébaud}

Stem cells were originally described by Till and McCulloch in 1960 (8). Their definition including the capability to form colonies (self-renew) and to differentiate into at least one other cell type still prevails. Embryonic stem cells (ESCs) represent the stem cell par excellence as they have extensive self-renewal capability and differentiate into all three germ layers. The best-described adult stem cells are hematopoietic stem cells. Their potential to reconstitute the entire hematopoiesis in the bone marrow is the basis for bone marrow transplantation in certain cancers for over 50 years. More recently, this approach has been used for the treatment of metabolic and immune diseases (9).

However, it is the discovery of the repair potential of mesenchymal stromal cells (MSCs) - until then considered as niche cells crucial for the regulation of hematopoietic stem cells-that ignited the use of cell therapies in regenerative medicine. Since the progress in stem cell biology has allowed the identification of numerous stem/progenitor cells, including endothelial progenitor cells (EPCs) for vascular repair and induced pluripotent stem cells (iPSCs) for genetic diseases, there is a strong rationale for harnessing the regenerative potential of these cells for neonatal lung diseases (Table 1).

\section{PLEIOTROPIC REPAIR CELLS TO STEM LUNG INJURY IN BPD Rationale}

BPD is a multifactorial disease interrupting normal lung growth. MSCs exert pleiotropic effects and release lung growth-promoting factors. As such, MSCs represent ideal therapeutic cells capable of stemming the multiple pathogenic mechanisms of BPD while also promoting lung growth, which is in stark contrast to currently used postnatal steroid therapy (10).

\section{Mesenchymal stromal cells}

Originally isolated from the bone marrow by Friedenstein as a rare population of plastic-adherent stromal cells ( 1 in 10,000 nucleated cells), MSCs have now been found to reside in all tissues (11). These plastic adherent cells were capable of forming single-cell colonies resembling fibroblastoid cells (colony-forming unit fibroblasts) and to differentiate into mesoderm-derived tissue. Because of different methods in the isolation and expansion, and different approaches to characterizing these cells between labs, the International Society for Cellular Therapy proposed minimal criteria to define human MSCs, including (i) adhesion to plastic, (ii) expression of the cell surface markers CD105, CD73, and CD90, and (iii) lack of CD 45, CD 34, CD14 or CD11b, CD79 $\alpha$ or CD19, and HLA-DR surface molecules expression, and (iv) differentiation to osteoblasts, adipocytes, and chondroblasts in vitro (12). While the definition of MSCs is still evolving, their ease of isolation and expansion, genetic stability, and pleiotropic effects make MSCs ideal repair cells, and thus the therapeutic potential of exogenous MSCs has been harnessed for the repair of various organ injuries, including the lung (13). In addition, MSC express HLA class I but not HLA class II and do not elicit alloreactive lymphocyte proliferation. Thus, MSCs are not inherently immunogenic, making allogeneic transplantation feasible, even to HLA-incompatible recipients.

\section{Proof-of-concept and preclinical studies in rodent models}

Bone marrow-derived MSCs exhibit lung-protective effects in oxygen-induced arrested alveolar and lung vascular development in neonatal rodents via single intratracheal or intravenous injection $(14,15)$. Rapidly, human umbilical cord tissue and cord blood were proposed as a clinically relevant source for MSC therapy. Interestingly, cord-derived MSCs may yield superior repair potential than the more widely used bone marrow MSCs (16-18). Furthermore, parabiosis experiments stressed the importance of the youth of the recipient environment and the cells, implicating great repair potential of perinatal cells in neonates (19). Accordingly, experiments in the neonatal rats exposed to hyperoxia demonstrated the therapeutic benefit in lung injury prevention and rescue, with safety up to 6 months post-treatment $(20,21)$.

\section{Mechanism of action}

The self-renewal and differentiation potential of stem cells formed the premise for studying their repair capabilities. Contrary to the original hypotheses, MSC engraftment and differentiation in the lung were consistently low (21). Thus, cell replacement does not seem important in the repair process. Instead, MSCs exert their beneficial effects through paracrine activity and cell-to-cell contact explaining their pleiotropic effects (reviewed in ref. (22)). Besides their potent immune-modulatory activities, MSCs also exert antiapoptotic, antioxidant, and antifibrotic effects. In addition, MSCs release numerous growth factors-including keratinocyte growth factor, epidermal growth factor, and angiogenic growth factors (23) - that independently have been shown to promote lung growth. Accordingly, in vitro and in vivo experiments

Table 1. Rationale approach to potential cell therapies based on disease mechanism

\begin{tabular}{|c|c|c|c|c|}
\hline Neonatal lung disease & Main disease mechanism & Cell therapy & Mechanism of action & Status \\
\hline BPD & Inflammation & MSC hAEC & Paracrine effect & $\begin{array}{l}\text {-Preclinical } \\
\text {-Phase I }\end{array}$ \\
\hline Pulmonary hypertension & Lung vascular hypoplasia/endothelial dysfunction & EPC & Paracrine effect & $\begin{array}{l}\text {-Preclinical } \\
\text {-Phase I }\end{array}$ \\
\hline Surfactant protein deficiency & Monogenetic mutation affecting a single-cell type & iPSC-derived AT2 & Cell replacement & -In vitro differentiation \\
\hline
\end{tabular}




\section{Stem cells for neonatal lung diseases $\quad$ Review}

with cell-free conditioned media derived from MSCs demonstrated lung-protective effects similar to whole-cell therapies $(14,15,21,23)$. Preconditioning in hyperoxia further enhanced the repair potential of MSC-derived conditioned media in the hyperoxic neonatal rat model (24). The recent realization that MSCs communicate and release bioactive compounds via extracellular vesicles (reviewed in ref. (25)) opens exciting new therapeutic avenues for cell therapies without the cell.

\section{Human amnion epithelial cells}

Human amnion epithelial cells (hAECs) represent another interesting perinatal tissue-derived cell product for the treatment of BPD. Intriguingly, hAECs isolated from the amniotic membrane after birth, share similar, mostly antiinflammatory properties with MSCs (26). Similar to MSCs, hAECs do not engraft and exert their therapeutic benefit through a paracrine activity. Preclinical studies have demonstrated the lung-protective effects of hAECs in neonatal rodents and fetal sheep (27-29).

Overall, these promising findings have prompted early phase clinical trials with MSCs and hAECs to test the feasibility and safety of these cells therapies in preterm infants.

\section{Early phase clinical trials}

A first phase I dose-escalation study enrolled nine extreme preterm neonates to receive a single intratracheal injection of allogeneic cord blood-derived MSCs within 5-14 days of life if still on mechanical ventilation (30). The procedure was feasible and no serious adverse events were reported. The recent follow-up study showed no transplantation-related adverse outcomes, including tumorigenicity. Infants treated with MSCs showed no adverse effects on growth, respiratory function (no asthma or requirement for continuous steroid/ bronchodilator treatment), and neurodevelopmental outcomes (including cerebral palsy, blindness, or developmental delay) at 2 years of age (31).

A phase I trial with hAECs just finished enrollment. In this trial, the safety and feasibility of a single dose of $1 \times 10^{6}$ cells/ $\mathrm{kg}$ of allogeneic hAECs intravenously was tested in six extreme preterm infants $\leq 28$ weeks gestation with established BPD (36 weeks corrected age, ongoing respiratory supporteither intubated or noninvasive respiratory support via nasal intermittent positive ventilation or continuous positive airway pressure therapy with mean/end expiratory pressure $>7 \mathrm{~cm}$ $\mathrm{H}_{2} \mathrm{O}$ and $\mathrm{FiO}_{2}=0.3-0.5$ ) (https://www.anzctr.org.au/Trial/ Registration/TrialReview.aspx?id = 365566).

While safety of these cells will likely be revealed, welldesigned phase II and III trials will need to demonstrate the therapeutic benefit. It will be critical to establish high-quality manufacturing processes to produce the most potent therapeutic product and identify reliable potency assays to predict as best as possible the efficacy of this product $(32,33)$. Indeed, variations in MSC products can make the interpretation of clinical trial results and ensuing systematic reviews and meta-analysis difficult.

\section{EPCS TO PROMOTE LUNG VASCULAR GROWTH AND ATTENUATE NEONATAL PH \\ Rationale}

Impaired lung growth and $\mathrm{PH}$ limits survival in a subset of infants with BPD and CDH. The anatomical reduction of the pulmonary vascular bed in these patients may render any new treatment strategy aiming at vasodilatation only-even so vasoconstriction might be present-obsolete. Blood vessel formation contributes to lung growth. EPCs contribute to vessel formation and may be altered in BPD/CDH. Consequently, supplementation of healthy EPCs may promote lung vascular growth and attenuate $\mathrm{PH}$.

\section{Endothelial progenitor cells}

Extensive work over the past two decades has highlighted the critical interactions between airways and blood vessels for normal lung development (34). The fact that combined abnormalities in the vasculature and airways occur in lung diseases suggested that (i) blood vessel formation is important for normal airway development; (ii) impaired signaling of angiogenic growth factors such as vascular endothelial growth factor leads to decreased lung growth and $\mathrm{PH}$; and (iii) enhancing angiogenesis via angiogenic growth factors promotes lung growth and attenuates $\mathrm{PH}$. These observations provided proof of concept for the crucial role of the lung vasculature in what was traditionally thought of as an airway disease and open new therapeutic avenues. These experiments also emphasized the importance of closely recapitulating the tightly orchestrated process of angiogenesis to warrant efficient and safe angiogenesis. This possibility was enabled by the discovery of circulating EPCs in adult human peripheral blood capable of postnatal neoangiogenesis in vivo (35).

Yet, controversy around the definition of EPCs, similar to what the field experienced with ES cells and MSCs, is a major limitation to decide which cell population to consider for cell therapy. Various methods exist for the isolation of EPCs and yield different type of cells. A recent consensus statement on nomenclature of EPCs suggests the use of precise terminology based on defining cellular phenotype and function (36). Endothelial colony-forming cells (ECFCs) and myeloid angiogenic cells (MACs) are examples of two distinct and well-defined cell types that have been considered EPCs: both promote vascular repair and may thus be considered for therapeutic purposes, although their mechanism of action appear to be different. ECFCs were originally identified by $\mathrm{Dr}$ Yoder's group in circulating adult and cord blood (37). Importantly, in contrast to MACs-which participate in angiogenesis but do not directly form the endothelial monolayer of new vessels and display various monocyte/ macrophage phenotypes and function-ECFCs are characterized by (i) robust proliferative potential, (ii) secondary and tertiary colony formation upon replating, and (iii) de novo blood vessel formation in vivo when transplanted into immunodeficient mice. 


\section{Proof of concept for the therapeutic potential of EPCs in experimental neonatal $\mathrm{PH}$}

Following the discovery of resident ECFCs in the adult rat lung (38), ECFCs were also identified in the developing human and rodent lung (39), as well as in human placenta (40). Interestingly, human fetal lung ECFCs exposed to hyperoxia in vitro and neonatal rat ECFCs isolated from hyperoxic injured lungs-mimicking $\mathrm{BPD}$ - proliferate less, have decreased clonogenic capacity, and form fewer capillarylike networks (41). Additional evidence supporting ECFC impairment associated with neonatal lung injury come from clinical studies. The number of cord blood ECFCs is significantly lower in preterm infants, which subsequently develop moderate or severe BPD $(42,43)$. Preterm ECFCs are also more susceptible to oxygen than term ECFCs (44). Data in $\mathrm{CDH}$ infants are more controversial: in one study, $\mathrm{CDH}$ infants had more high proliferative ECFCs in their cord blood compared with controls (45). Conversely, another study reported impaired cord blood ECFC self-renewal, in vivo vasculogenesis, and response to vascular endothelial growth factor from $\mathrm{CDH}$ infants compared with healthy term infants (46). Together with other studies using EPCs as biomarkers for neonatal complications, these observations highlight the need for larger sample sizes on clearly defined EPCs to provide more robust data (47). Overall, these findings provided sufficient rationale to test the therapeutic potential of ECFCs and MACs in animal models.

Intravenous infusion of human cord blood-derived ECFCs into immune-deficient rats and mice exposed to hyperoxia promote lung vascular growth, prevent and restore alveolar injury, and attenuate $\mathrm{PH}$ with safety at 10 months posttreatment (41). Intriguingly and similar to observations with MSCs, low ECFC engraftment and the protective effect of cellfree ECFC-derived conditioned media (41) suggest that ECFCs exert their therapeutic benefit via a paracrine effect. In a bleomycin-induced neonatal lung injury model, ECFCderived conditioned media had no effect on lung growth but attenuated PH (48). Unlike MSCs, ECFC may be immunogenic, hampering the possibility of non-major histocompatibility complex-matched allogeneic transplantation. Thus, the paracrine mode of action of ECFCs may open exciting therapeutic avenues for cell-free, allogeneic, off-the-shelf proangiogenic therapies.

Circulating, lung and bone marrow-derived MACs are reduced in neonatal mice exposed to hyperoxia (49). Conversely, hyperoxic adult mice did not display alveolar damage and had increased circulating and lung MACs, implying that decreased proangiogenic cells may contribute to the arrested lung growth seen in the neonatal animals. Infusion of bone marrow-derived MACs restored alveolar and lung vascular growth in hyperoxic neonatal mice (50).

Well-designed preclinical studies using small and large animal models are required to provide a solid basis for the development of safe proangiogenic cell products that can be translated into the clinic.

\section{IPSCS FOR MONOGENETIC LUNG DISEASES}

\section{Rationale}

Surfactant protein deficiencies are devastating lung diseases that can be lethal or lead to interstitial lung diseases (7). These genetic lung disorders affect a single gene in a single cell type. Patient-specific iPSCs can be derived from skin fibroblasts or blood cells and cord, and then differentiated into any cell type, including surfactant producing alveolar epithelial type 2 (AT2) cells. Thus, iPSCs are ideally positioned for disease modeling, genome editing strategies, and cell replacement therapies.

\section{Induced pluripotent stem cells}

iPSCs are one of the most exciting breakthroughs of the past decade. Somatic cell nuclear transfer experiments by $\mathrm{Dr}$ Gurdon in the 1960s demonstrated that fully differentiated somatic cells retained pluripotent abilities (51). It took Shinya Yamanaka's identification of four transcription factors (Oct3/4, Sox2, Klf4, and c-Myc) to reprogram fully differentiated somatic cells into pluripotent stem cells to realize that a new potential therapeutic strategy for diseases was now available $(52,53)$. These human (h)iPSCs possess desirable characteristics for use in cell therapies. Similar to ESCs, hiPSCs have the potential to become any cell type in the body. However, as hiPSCs can be generated from each patient, they represent an autologous source of cells that overcomes the likelihood of immune-mediated rejection. An unlimited number of cells can be generated from hiPSCs as they can be maintained and expanded in vitro. hiPSC clones can also be manipulated by gene editing technologies to correct DNA mutations. And unlike ESCs, hiPSCs are not associated with the controversies and ethical considerations commonly linked with using ESCs.

\section{Proof of concept that iPSCs can be differentiated into alveolar epithelial cells}

Early studies differentiated ESCs into AT2 cells within 11 days of using a two-step protocol into definitive endoderm by activin or A549-conditioned medium as a precursor to lung epithelial cells (54). Wnt3 was identified as endoderm differentiation-promoting factor, and fibroblast growth factor 2 seemed to enhance maturation into alveolar epithelial cells. Endotracheal injection of ESCs into preterm mouse lung suggested that recapitulation of development enhance derivation of an enriched population of lung-like cells. Novel tools $(55,56)$ and the development of more complex step-wise protocols to recapitulate endoderm differentiation and lung development in a dish can now facilitate the successful generation of conducting airway epithelial cells (57) useful for cystic fibrosis and also distal alveolar epithelial cells $(58,59)$. Current refinements of differentiation protocols include the generation of three-dimensional organoid structures to more reliably recapitulate the lung environment (60). It is now possible to generate hiPSC-derived lung bud organoids containing mesoderm and pulmonary endoderm and develop into branching airway and early alveolar structures after xenotransplantation and in Matrigel three-dimensional 
culture mimicking the second trimester of human gestation (61). This organoid approach can be used for synthesizing patient-specific lung tissue to model any human lung disease with the potential for high-throughput drug screening to identify novel therapies (62).

\section{Unprecedented therapeutic possibilities for monogenetic lung diseases}

The potential for disease modeling and testing of therapeutic strategies has been elegantly demonstrated for cystic fibrosis. Using zinc-finger nucleases (63) or CRISPR/Cas9 (ref. (64)) in hiPSCs reprogrammed from cystic fibrosis patients, mutations in the CFTR gene were corrected by gene editing. The hiPSCs containing the newly corrected CFTR gene were subsequently differentiated into airway epithelial cells in which CFTR ion transport function was fully restored $(63,64)$. More recently, functional proximal airway organoids were generated by directed differentiation of patient-specific iPSCs that displayed defects in forskolin-induced swelling and could be rescued by gene editing to correct the disease mutation (65).

These studies provide proof of concept that patient hiPSC generation combined with gene editing is a feasible therapeutic strategy for genetic lung diseases such as cystic fibrosis, and that this strategy can likely be applied to lung diseases such as genetic surfactant disorders. For example, in a patient carrying the most common mutation in SP-B deficiency (121 ins2), gene editing is required to delete the two inserted nucleotides in codon 121 of the SFTPB gene by homologous recombination and restore the correct reading frame. The hiPSCs containing the corrected SFTPB gene can then be differentiated into AT2 cells. Once expanded, these cells would be available for cell therapy.

An important question for therapies is the dosage required to demonstrate a therapeutic benefit for the patient. AT2 cells comprise $7 \%$ of the total alveolar surface and $16 \%$ of all parenchymal cells $(66,67)$. It is unlikely that cell therapy could restore the full percentage of AT2 cells in the parenchyma because of poor engraftment and immune-mediated rejection. However, for individuals with SFTPB mutations, it is possible that cell therapy only requires a partial restoration of SPB to $20-25 \%$ of wild-type levels. In SP-B mouse models, symptoms of surfactant deficiency only appear when SP-B falls below $20-25 \%$ of wild-type levels (68). Previous studies have already demonstrated the effectiveness of AT2-derived iPSC therapy in an animal model of lung fibrosis (69). However, the translation of these findings into a therapy for humans remains difficult. Other strategies, such as in vivo genome editing (70), are being considered and may yield superior results as a separate approach or in combination with cell therapy.

\section{Important hurdles to overcome}

A first clinical trial involving hiPSC-derived retinal epithelial cells for age-related macular degeneration showed no tissue rejection and no adverse events, but visual acuity had not changed 1 year following the surgery. Although hiPSCs represent a promising new strategy for ameliorating genetic diseases with limited treatment options, hiPSC therapy is still in its infancy and a number of caveats must be addressed before hiPSCs can be considered a viable form of therapy for patients with genetic conditions of surfactant deficiency. The transcription factor-mediated process to reprogram somatic cells into hiPSCs can alter the methylation status of the hiPSCs compared with ESCs (71). It is unknown how this difference in methylation affects the downstream applications of hiPSCs. The original methylation status of the somatic cell is observed in the reprogrammed hiPSCs. Normal genetic variation between individuals also results in heterogeneity of hiPSC populations following reprogramming (72). This means that even though reprogramming conditions are kept consistent between different cell lines, hiPSCs will be heterogeneous because of their differences in methylation patterns and their inherent genetic variation. This heterogeneity can result in unknown responses when differentiating hiPSCs into AT2 cells. Furthermore, human pluripotent stem cells (including ES and iPSC) accumulate dominant-negative mutations in tumor suppressor genes over time, presenting a cancer risk to patients if human pluripotent cells are used as a therapeutic (73). Another factor to consider is that the current differentiation protocols to generate AT2 cells from hiPSCs can be inefficient, still use xenogenic agents, and there are concerns about how well the hiPSC-derived AT2 cells resemble naturally occurring mature and functional AT2 cells (74). Finally, even though hiPSC-derived cells represent an autologous cell population, and should not generate an immune response, animal studies have demonstrated that autologous iPSC-derived cells can be immunogenic (75). The immunogenicity as well as tumorigenicity of differentiated cells or undifferentiated hiPSCs should always be assessed before using these cells as a therapy.

hiPSCs represent a novel and exciting therapeutic strategy for monogenetic disorders of the lung. While in vitro disease modeling using hiPSCs may allow the development of novel therapies in the near future, much more needs to be learned about hiPSCs and potential risks must be addressed before these cells are used in patients.

\section{CONCLUSION}

Cell therapies may represent a paradigm shift in the treatment of neonatal lung diseases. Promising preclinical studies with MSC and hAECS have already led to early phase clinical trials in infants at risk of developing BPD. ECFCs and MACs, interesting proangiogenic cells for intractable $\mathrm{PH}$ in infants with $\mathrm{CDH}$ and vascular BPD phenotype, have passed the proof-of-concept stage and lessons learned from MSCs and hAECS may hasten the development of theses cells into a safe clinical-grade product. hiPSCs hold tremendous promise for curing monogenetic lung diseases either through disease modeling and drug screening, as exemplified by in vitro studies, or through direct cell replacement. While each of these cells are at different stages of clinical translation, much more needs to be learned about their biology to develop disease-specific and safe cell products. The manufacturing 
process will be critical to obtain consistent, high-quality cell product with predictable activity in vivo. Ultimately, the successful clinical translation can only be based on the above and methodologically robust preclinical studies that provide a strong rationale to test relevant cell product in patients (76).

\section{STATEMENT OF FINANCIAL SUPPORT AND DISCLOSURE}

B.T. is supported by the Canadian Institute of Health Research, Canadian Thoracic Society, Ontario Institute for Regenerative Medicine, and the Children's Hospital of Eastern Ontario foundation.

Disclosure: The authors declare no conflict of interest.

\section{REFERENCES}

1. Owen LS, Manley BJ, Davis PG, Doyle LW. The evolution of modern respiratory care for preterm infants. Lancet 2017;389:1649-59.

2. Stoll BJ, Hansen NI, Bell EF, et al. Trends in care practices, morbidity, and mortality of extremely preterm neonates, 1993-2012. JAMA 2015;314:1039-51.

3. Abman SH. Bronchopulmonary dysplasia: "a vascular hypothesis". Am J Respir Crit Care Med 2001;164:1755-6.

4. Khemani E, McElhinney DB, Rhein L, et al. Pulmonary artery hypertension in formerly premature infants with bronchopulmonary dysplasia: clinical features and outcomes in the surfactant era. Pediatrics 2007;120:1260-9.

5. Kinsella JP, Ivy DD, Abman SH. Pulmonary vasodilator therapy in congenital diaphragmatic hernia: acute, late, and chronic pulmonary hypertension. Semin Perinatol 2005;29:123-8.

6. Lusk LA, Wai KC, Moon-Grady AJ, Steurer MA, Keller RL. Persistence of pulmonary hypertension by echocardiography predicts short-term outcomes in congenital diaphragmatic hernia. J Pediatr 2015;166: 251-56 and 251.

7. Nogee LM. Interstitial lung disease in newborns. Semin Fetal Neonatal Med 2017;22:227-33.

8. McCulloch EA, Till JE. The radiation sensitivity of normal mouse bone marrow cells, determined by quantitative marrow transplantation into irradiated mice. Radiat Res 1960;13:115-25.

9. Atkins H, Freedman MS. Immunoablation and aHSCT for aggressive multiple sclerosis-Authors' reply. Lancet 2017;389:908.

10. le Cras TD, Markham NE, Morris KG, Ahrens CR, McMurtry IF, Abman $\mathrm{SH}$. Neonatal dexamethasone treatment increases the risk for pulmonary hypertension in adult rats. Am J Physiol Lung Cell Mol Physiol 2000;278: L822-829.

11. Friedenstein AJ, Gorskaja JF, Kulagina NN. Fibroblast precursors in normal and irradiated mouse hematopoietic organs. Exp Hematol 1976;4: 267-74.

12. Dominici M, Le Blanc K, Mueller I, et al. Minimal criteria for defining multipotent mesenchymal stromal cells. The International Society for Cellular Therapy position statement. Cytotherapy 2006;8:315-7.

13. Wagner DE, Cardoso WV, Gilpin SE, et al. An Official American Thoracic Society Workshop Report 2015. Stem cells and cell therapies in lung biology and diseases. Ann Am Thorac Soc 2016;13:S259-78.

14. Aslam M, Baveja R, Liang OD, et al. Bone marrow stromal cells attenuate lung injury in a murine model of neonatal chronic lung disease. Am J Respir Crit Care Med 2009;180:1122-30.

15. van Haaften T, Byrne R, Bonnet S, et al. Airway delivery of mesenchymal stem cells prevents arrested alveolar growth in neonatal lung injury in rats. Am J Respir Crit Care Med 2009;180:1131-42.

16. Hsieh JY, Wang HW, Chang SJ, et al. Mesenchymal stem cells from human umbilical cord express preferentially secreted factors related to neuroprotection, neurogenesis, and angiogenesis. PLoS ONE 2013;8:e72604.

17. Jin HJ, Bae YK, Kim M, et al. Comparative analysis of human mesenchymal stem cells from bone marrow, adipose tissue, and umbilical cord blood as sources of cell therapy. Intern J Mol Sci 2013;14: 17986-8001.
18. Yannarelli G, Dayan V, Pacienza N, Lee CJ, Medin J, Keating A. Human umbilical cord perivascular cells exhibit enhanced cardiomyocyte reprogramming and cardiac function after experimental acute myocardial infarction. Cell Transplant 2013;22:1651-66.

19. Conboy IM, Conboy MJ, Wagers AJ, Girma ER, Weissman IL, Rando TA. Rejuvenation of aged progenitor cells by exposure to a young systemic environment. Nature 2005;433:760-4.

20. Chang YS, Choi SJ, Ahn SY, et al. Timing of umbilical cord blood derived mesenchymal stem cells transplantation determines the rapeutic efficacy in the neonatal hyperoxic lung injury. PLoS ONE 2013;8: e52419.

21. Pierro M, Ionescu L, Montemurro T, et al. Short-term, long-term and paracrine effect of human umbilical cord-derived stem cells in lung injury prevention and repair in experimental bronchopulmonary dysplasia. Thorax 2013;68 (5): 475-84.

22. Fung ME, Thebaud B. Stem cell-based therapy for neonatal lung disease: it is in the juice. Pediatr Res 2014;75:2-7.

23. Chang YS, Ahn SY, Jeon HB, et al. Critical role of vascular endothelial growth factor secreted by mesenchymal stem cells in hyperoxic lung injury. Am J Respir Cell Mol Biol 2014;51:391-9.

24. Waszak P, Alphonse R, Vadivel A, Ionescu L, Eaton F, Thebaud B. Preconditioning enhances the paracrine effect of mesenchymal stem cells in preventing oxygen-induced neonatal lung injury in rats. Stem Cells Dev 2012;21:2789-97.

25. Kourembanas S. Exosomes: vehicles of intercellular signaling, biomarkers, and vectors of cell therapy. Annu Rev Physiol 2015;77:13-27.

26. Ilancheran S, Michalska A, Peh G, Wallace EM, Pera M, Manuelpillai U. Stem cells derived from human fetal membranes display multilineage differentiation potential. Biol Reprod 2007;77:577-88.

27. Vosdoganes P, Hodges RJ, Lim R, et al. Human amnion epithelial cells as a treatment for inflammation-induced fetal lung injury in sheep. Am J Obstet Gynecol 2011;205:156 and 126-33.

28. Vosdoganes P, Lim R, Koulaeva E, et al. Human amnion epithelial cells modulate hyperoxia-induced neonatal lung injury in mice. Cytotherapy 2013;15:1021-9.

29. Vosdoganes P, Wallace EM, Chan ST, Acharya R, Moss TJ, Lim R. Human amnion epithelial cells repair established lung injury. Cell Transplant 2013;22:1337-49.

30. Chang YS, Ahn SY, Yoo HS, et al. Mesenchymal stem cells for bronchopulmonary dysplasia: phase 1 dose-escalation clinical trial. J Pediatr 2014;164:966-972 and 966.

31. Ahn SY, Chang YS, Kim JH, Sung SI, Park WS. Two-year follow-up outcomes of premature infants enrolled in the phase I trial of mesenchymal stem cells transplantation for bronchopulmonary dysplasia. J Pediatr 2017;185:49-54.

32. Boregowda SV, Phinney DG. Quantifiable metrics for predicting MSC therapeutic efficacy. J Stem Cell Res Ther 2016;6:6. pii: 365.

33. Martin I, De Boer J, Sensebe LMSC Committee of the International Society for Cellular Therapy. A relativity concept in mesenchymal stromal cell manufacturing. Cytotherapy 2016;18:613-20.

34. Thebaud B, Abman SH. Bronchopulmonary dysplasia: where have all the vessels gone? Roles of angiogenic growth factors in chronic lung disease. Am J Respir Crit Care Med 2007;175:978-85.

35. Asahara T, Murohara T, Sullivan A, et al. Isolation of putative progenitor endothelial cells for angiogenesis. Science 1997;275:964-7.

36. Medina RJ, Barber CL, Sabatier F, et al. Endothelial progenitors: a consensus statement on nomenclature. Stem Cells Transl Med 2017;6:1316-20.

37. Ingram DA, Mead LE, Tanaka H, et al. Identification of a novel hierarchy of endothelial progenitor cells using human peripheral and umbilical cord blood. Blood 2004;104:2752-60.

38. Alvarez DF, Huang L, King JA, Elzarrad MK, Yoder MC, Stevens T. Lung microvascular endothelium is enriched with progenitor cells that exhibit vasculogenic capacity. Am J Physiol Lung Cell Mol Physiol 2008;294: L419-430.

39. Alphonse RS, Vadivel A, Zhong S, et al. The isolation and culture of endothelial colony-forming cells from human and rat lungs. Nature Protoc 2015;10:1697-708. 
40. Solomon I, O'Reilly M, Ionescu L, et al. Functional differences between placental micro- and macrovascular endothelial colony-forming cells. Stem Cells Transl Med 2016;5:291-300.

41. Alphonse RS, Vadivel A, Fung M, et al. Existence, functional impairment, and lung repair potential of endothelial colony-forming cells in oxygeninduced arrested alveolar growth. Circulation 2014;129:2144-57.

42. Baker CD, Balasubramaniam V, Mourani PM, et al. Cord blood angiogenic progenitor cells are decreased in bronchopulmonary dysplasia. Eur Respir J 2012;40:1516-22.

43. Borghesi A, Massa M, Campanelli R, et al. Circulating endothelial progenitor cells in preterm infants with bronchopulmonary dysplasia. Am J Respir Crit Care Med 2009;180:540-6.

44. Baker CD, Ryan SL, Ingram DA, et al. Endothelial colony-forming cells from preterm infants are increased and more susceptible to hyperoxia. Am J Respir Crit Care Med 2009;180:454-61.

45. Baker CD, Black CP, Ryan SL, Balasubramaniam V, Abman SH. Cord blood endothelial colony-forming cells from newborns with congenital diaphragmatic hernia. J Pediatr 2013;163:905-7.

46. Fujinaga H, Fujinaga H, Watanabe N, et al. Cord blood-derived endothelial colony-forming cell function is disrupted in congenital diaphragmatic hernia. Am J Physiol Lung Cell Mol Physiol 2016;310:L1143-1154.

47. Bertagnolli M, Nuyt AM, Thebaud B, Luu TM. Endothelial progenitor cells as prognostic markers of preterm birth-associated complications. Stem Cells Transl Med 2017;6 (1): 7-13.

48. Baker CD, Seedorf GJ, Wisniewski BL, et al. Endothelial colony-forming cell conditioned media promote angiogenesis in vitro and prevent pulmonary hypertension in experimental bronchopulmonary dysplasia. Am J Physiol Lung Cell Mol Physiol 2013;305:L73-81.

49. Balasubramaniam V, Mervis CF, Maxey AM, Markham NE, Abman SH. Hyperoxia reduces bone marrow, circulating, and lung endothelial progenitor cells in the developing lung: implications for the pathogenesis of bronchopulmonary dysplasia. Am J Physiol Lung Cell Mol Physiol 2007;292:L1073-1084.

50. Balasubramaniam V, Ryan SL, Seedorf GJ, et al. Bone marrow-derived angiogenic cells restore lung alveolar and vascular structure after neonatal hyperoxia in infant mice. Am J Physiol Lung Cell Mol Physiol 2010;298: L315-323.

51. Gurdon JB. The developmental capacity of nuclei taken from intestinal epithelium cells of feeding tadpoles. J Embryol Exp Morphol 1962;10:622-40.

52. Takahashi K, Tanabe K, Ohnuki M, et al. Induction of pluripotent stem cells from adult human fibroblasts by defined factors. Cell 2007;131:861-72.

53. Takahashi K, Yamanaka S. Induction of pluripotent stem cells from mouse embryonic and adult fibroblast cultures by defined factors. Cell 2006;126:663-76.

54. Roszell B, Mondrinos MJ, Seaton A, et al. Efficient derivation of alveolar type II cells from embryonic stem cells for in vivo application. Tissue Eng Part A 2009;15:3351-65.

55. Hawkins F, Kramer P, Jacob A, et al. Prospective isolation of NKX2-1expressing human lung progenitors derived from pluripotent stem cells. J Clin Invest 2017;127:2277-94.

56. Longmire TA, Ikonomou L, Hawkins F, et al. Efficient derivation of purified lung and thyroid progenitors from embryonic stem cells. Cell Stem Cell 2012;10:398-411.

57. Mou H, Zhao R, Sherwood R, et al. Generation of multipotent lung and airway progenitors from mouse ESCs and patient-specific cystic fibrosis iPSCs. Cell Stem Cell 2012;10:385-97.
58. Ghaedi M, Calle EA, Mendez JJ, et al. Human iPS cell-derived alveolar epithelium repopulates lung extracellular matrix. J Clin Invest 2013;123: 4950-62.

59. Huang SX, Islam MN, O'Neill J, et al. Efficient generation of lung and airway epithelial cells from human pluripotent stem cells. Nat Biotechnol 2014;32:84-91.

60. Barkauskas CE, Chung MI, Fioret B, Gao X, Katsura H, Hogan BL. Lung organoids: current uses and future promise. Development 2017;144: 986-97.

61. Chen YW, Huang SX, de Carvalho A, et al. A three-dimensional model of human lung development and disease from pluripotent stem cells. Nat Cell Biol 2017;19:542-9.

62. Wilkinson DC, Alva-Ornelas JA, Sucre JM, et al. Development of a three-dimensional bioengineering technology to generate lung tissue for personalized disease modeling. Stem Cells Transl Med 2017;6: 622-33.

63. Crane AM, Kramer P, Bui JH, et al. Targeted correction and restored function of the CFTR gene in cystic fibrosis induced pluripotent stem cells. Stem Cell Rep 2015;4:569-77.

64. Firth AL, Menon T, Parker GS, et al. Functional gene correction for cystic fibrosis in lung epithelial cells generated from patient iPSCs. Cell Rep 2015;12:1385-90.

65. McCauley KB, Hawkins F, Serra M, Thomas DC, Jacob A, Kotton DN. Efficient derivation of functional human airway epithelium from pluripotent stem cells via temporal regulation of Wnt signaling. Cell Stem Cell 2017;20:844-857 e846.

66. Crapo JD, Barry BE, Gehr P, Bachofen M, Weibel ER. Cell number and cell characteristics of the normal human lung. Am Rev Respir Dis 1982;126:332-7.

67. Crapo JD, Barry BE, Gehr P, Bachofen M, Weibel ER. Cell number and cell characteristics of the normal human lung. Am Rev Respir Dis 1982;125:740-5.

68. Melton KR, Nesslein LL, Ikegami M, et al. SP-B deficiency causes respiratory failure in adult mice. Am J Physiol Lung Cell Mol Physiol 2003;285:L543-549.

69. Soh BS, Zheng D, Li Yeo JS, et al. CD166pos subpopulation from differentiated human ES and iPS cells support repair of acute lung injury. Mol Ther 2012;20:2335-46.

70. Mahiny AJ, Dewerth A, Mays LE, et al. In vivo genome editing using nuclease-encoding mRNA corrects SP-B deficiency. Nat Biotechnol 2015;33:584-6.

71. Mandai M, Watanabe A, Kurimoto Y, et al. Autologous induced stemcell-derived retinal cells for macular degeneration. N Engl J Med 2017;376:1038-46.

72. Kilpinen H, Goncalves A, Leha A, et al. Common genetic variation drives molecular heterogeneity in human iPSCs. Nature 2017;546:370-5.

73. Merkle FT, Ghosh S, Kamitaki N, et al. Human pluripotent stem cells recurrently acquire and expand dominant negative P53 mutations. Nature 2017;545:229-33.

74. Beers MF, Moodley Y. When is an alveolar type 2 cell an alveolar type 2 cell? A conundrum for lung stem cell biology and regenerative medicine. Am J Respir Cell Mol Biol 2017;57:18-27.

75. Zhao T, Zhang ZN, Rong Z, Xu Y. Immunogenicity of induced pluripotent stem cells. Nature 2011;474:212-5.

76. Daley GQ. Polar extremes in the clinical use of stem cells. N Engl J Med 2017;376:1075-7. 